\title{
Microfluidic Device Control System Based on Segmented Temperature Sensor
}

\author{
Anlin Xu (iD) and Ping Li \\ Wuxi Institute of Technology, Wuxi 214121, China \\ Correspondence should be addressed to Anlin Xu; xuanlin@wxit.edu.cn \\ Received 29 March 2021; Revised 29 April 2021; Accepted 5 May 2021; Published 19 May 2021
}

Academic Editor: Fazlullah Khan

Copyright (c) 2021 Anlin Xu and Ping Li. This is an open access article distributed under the Creative Commons Attribution License, which permits unrestricted use, distribution, and reproduction in any medium, provided the original work is properly cited.

\begin{abstract}
Microfluidic technology refers to the technique of controlling the flow, mass transfer, and heat transfer of a fluid with a volume of picoliter to nanoliter in a low-dimensional channel structure with at least one dimension of micron or even nanometer scale. It is widely used in biochemical analysis, immunity, minimally invasive surgery, and environmental monitoring. This paper proposes a microfluidic device based on a segmented temperature sensor. This device can be used for segmental temperature measurement and controlling the temperature of the solution in the microchannel of a glass microfluidic chip. The device is based on a transparent indium tin oxide film glass as a heating element. It adopts a temperature control platform of a proportional-integralderivative control algorithm. The system uses a charged coupled device camera, a fluorescence microscope, and an image acquisition card to form a noncontact fluorescent indicator temperature measuring device. The device measures the temperature distribution of the microfluid space with time and controls the microfluidics. Moreover, the device has the advantages of simple structure, low cost, and convenient operation.
\end{abstract}

\section{Introduction}

Temperature is an essential parameter in biochemical research, such as bioorganic synthesis, polymerase chain reaction, and gene mutation detection. With the increasing application of microfluidic chip systems in the biochemical analysis [1], accurate measurement of temperature in microfluidic systems and control is becoming very critical. Since the volume of the fluid in the microfluidic system is generally on $\mathrm{pL}$ to $\mathrm{nL}$, the contact temperature measurement method disturbs the microfluid and changes its actual temperature. It is more difficult to measure the temperature distribution in the two-dimensional space in the flow field [2]. It has been reported in the literature that the temperature measuring components are directly integrated on the microfluidic chip. The size of the temperature measuring component can be on the order of micrometer $(\mu \mathrm{m})$. However, this method is challenging to achieve in a nonprofessional micromachining laboratory. The noncontact temperature measurement method is more suitable for microfluidic measurement. It is reported in the literature that the measurement accuracy of thermochromic liquid crystal temperature measurement technology can reach $011^{\circ} \mathrm{C}$. However, liquid crystal particles of $10-20 \mu \mathrm{m}$ size can cause shallow channel blockage, and the measurement range is narrow. The integrated heating method that directly integrates the heating element and the temperature-measuring element on the microchip by using the micromachining technology has the advantages of small volume, fast temperature response speed, and high control precision, but it also has the disadvantage of processing difficulty $[3,4]$. To measure temperature in a microfluidic chip, a general technique is the resistance temperature detector (RTD), a simple solution requiring minimal external equipment. RTDs can quickly fabricate and are suitable for steady-state temperature monitoring [5]. The response time of RTD is slow due to the heat transfer between the sample fluid and sensor. In addition, segmented droplets are often smaller than the sensor footprint required for RTD operation, which results in significant error. To measure the temperature of 
the microfluidic model in situ, methods based on fluorescence intensity ratio (FIR) are widely applied. The quantum yield of some fluorescence dyes such as rhodamine B is temperature-dependent, allowing spatial temperature distribution to be measured with less than $3.5^{\circ} \mathrm{C}$ uncertainty [6]. To increase accuracy, fluorescent systems with ultralow temperature coefficients are required. This method is more suitable for glass-based materials but is not well suited with porous chip materials such as polydimethylsiloxane (PDMS) because dye elements tend to diffuse with channel walls, creating nonnatural fluorescent intensities. Driven by the widespread use of PDMS for microfluidic chip fabrication and the high spatial resolution of temperature measurements enabled by rhodamine $\mathrm{B}$, several techniques have been developed to overcome such challenges [7]. Other molecular probes, such as fluorescein, are temperature-independent in their fluorescent intensity. However, their $\mathrm{pH}$ is temperature-dependent, which could measure temperature if a pH-temperature sensitive buffer is provided. Generally, temperature probe (CdSe)ZnS quantum dot is better since its emission and absorption spectra can be tuned during growth, but synthesis and fabrication into a usable form such as coated beads are costly. The primary source of error for all FIR-based methods is the nonuniform local probe concentration and fluctuation in excitation intensity. By employing two different dyes with separate emission spectra and two simultaneously operating cameras, the temperature can be related to the ratio between the two emission spectra, unaffected by concentration and source fluctuation. If picosecond photo counting equipment is available, fluorescence lifetime (FL) can be used to measure temperature instead $[8,9]$.

Like the FIR and FL methods, cholesteric liquid crystals (TLC) are applied to quantify temperature at $\pm 0.1^{\circ} \mathrm{C}$ accuracy. It provides high accuracy results from drastic hue change within a range of $1^{\circ} \mathrm{C}-2^{\circ} \mathrm{C}$. However, instead of high accuracy, the high viscosity and large bead size after necessary encapsulation have restricted the use of TLC in microfluidic devices. In this manuscript, we present a microfluidic device control system that is based on a segmented temperature sensor [10]. The significant contributions of the manuscript are as follows:

(i) In this paper, a microfluidic device for microfluidic temperature measurement and temperature control in a microfluidic chip system with a simple structure, low cost, and good performance is proposed.

(ii) The temperature measurement uses fluorescent dye as an indicator to realize the space of microfluid temperature.

(iii) The device has a simple structure, small volume, small heat capacity, fast temperature rise and fall, accurate temperature control, and a stable dynamic process.

The rest of the paper is organized as follows: In Section 2, a microfluidic device control system is discussed. In Section 3 , system composition and operation are discussed, followed by the control system of the microfluidic device based on the segmented temperature sensor in Section 4. Finally, the paper is concluded in Section 5.

\section{Microfluidic Device Control System}

2.1. Microfluidic Technology. Microfluidics is the science of the system. It uses a few tens to hundreds of micrometers of tubing to process or manipulate tiny amounts (10-9 to $10-18$ liters, 1 cubic millimeter to 1 cubic micrometer) of fluid and pass it through. The technology of mass and heat transfer can be widely used in many fields such as biochemical analysis, immunoassay, minimally invasive surgery, and environmental monitoring. The initial microfluidic technology was used for microfluidic device analysis. Microfluidics provides many valuable functions for analysis: high precision and high sensitivity separation and detection using very few samples and reagents, low cost, short analysis time, and small footprint of analytical equipment. Microfluidics takes advantage of its most apparent features-small size and the use of less obvious microchannel fluids, such as laminar flow. It essentially provides the ability to control molecules in space and time [11] centrally. The primary contents of microfluidic technology include the design and manufacture of microchannel structures, driving of micro-nanoscale fluids, and integration and packaging of microfluidic devices and systems.

2.2. Microchannel Structure and Design. The fluid in the microfluidic system needs to flow in a certain way in the microchannel of a specific size and structure to achieve heat transfer, mass transfer, and momentum transfer. Therefore, the microchannel is the core of the microfluidic system. Channels with larger dimensions (features larger than $100 \mathrm{~m}$ ), simple structures, and functions can be prepared with capillaries. In contrast, channels with small dimensions and complex structures need to be prepared using specific materials and specific processes $[12,13]$.

The microchannel consists of the main channel, an auxiliary channel (side stream channel), and an output VI. When multiphase fluids need to be input in the main channel, different fluids need to be introduced from different population channels and different outlet channels, and then different fluids processed by the main channel need to be exported. The inlet and outlet sections can be designed as a "T" type, a "Y" type mocking, or a fan-shaped structure. The main channel is the main space for separation, mixing, and the reaction of fluids $[14,15]$. It is the central part of the function of microfluidic devices. The structure and size should be carefully designed according to the specific functions to be realized by the device. The simplest channel is a flat straight channel. When necessary to cause adequate mixing between different fluids, the main channel can be designed as a two-dimensional curved type, a two-dimensional polygonal line type, a three-dimensional polygonal line type, or a more complicated three-dimensional structure [16]. Compared with the two-dimensional structure, the three-dimensional structure can generate a strong vortex, making the mixing faster and more efficient, but the 
manufacturing is more complicated. The wall or bottom of the main channel can also be designed with diagonal ribs, chutes, or herringbone grooves, causing anisotropic flow resistance and turbulence to enhance mixing [17, 18]. Under low Reynolds number conditions, the addition of ordered obstacles to the main channel can be used to efficiently separate particles of different sizes dispersed in a liquid. Auxiliary channels (lateral flow channels) are typically used to achieve hydraulic focusing and flow control of the fluid [18]. Figures 1(a)-1(c) represent the different types of channels, i.e., flat straight channel, two-dimensional curved main channel, and two-dimensional broken line main channel.

\subsection{Microchannel Development}

2.3.1. Design Materials. The microchannel structure can be prepared from different materials such as silicon, glass, and high molecular polymers. Monocrystalline silicon wafers are the basic materials of the integrated circuit (IC) industry and are widely used in semiconductor devices and ICs. The process of preparing microchannels on a single crystal silicon wafer is generally fully compatible with microfabrication techniques [19]. The prepared microfluidic device can be easily integrated with microelectronic devices. Ordinary glass based on silica is a low-cost, insulating material that is transparent to visible light. It is used to prepare microchannels for easy observation of the flow state in the channel and at the same time it can achieve adequate bonding with materials such as organic polymers and silicon to prevent fluid leakage. Compared to silicon wafers and glass, organic polymer materials are easier to process and prepare and can produce complex channel structures. The organic polymer materials that can be used to prepare the microchannels are mainly polycarbonate (PC), polymethyl methacrylate, polystyrene (PS), polyethylene terephthalate, polyvinyl chloride (PVC), polyethylene (PE), and polydimethylsiloxane (PDMS). PDMS has good elasticity and can be used for preparing special devices requiring large deformation, such as micro-gas pumps and microvalves. However, organic materials have poor thermal conductivity, high-temperature resistance, and inconsistent processing techniques with micromanufacturing processes, limiting their application in microfluidic technology [20, 21].

2.3.2. Processing and Preparation. The processing of microchannels on silicon and glass materials mainly uses photolithography and etching techniques to precisely control the shape, size, and position of the microchannels and simultaneously forms patterns on the entire chip surface that is suitable for mass production. With the advancement of the lithography process, the precision of lithography is continuously improved, and the size of the prepared graphic features is continuously reduced. Nanoscale lithography can be used to prepare microchannels with feature sizes less than $500 \mathrm{~nm}$, enabling control and application of nanoscale fluids. However, the lithography process is complicated, requires expensive exposure and etching equipment, and has high cost and low output. At the same time, photolithography and etching are challenging to prepare microchannels of threedimensional structure. The processing methods of polymer materials are quite different, usually done by soft lithography. The graphic master can be prepared by printing, ordinary lithography, or electron beam. The pattern transfer and copying can be realized by embossing [22], molding core 61 , and printing $\mathrm{m} 1$. The soft lithography technology ' 243 can not only manufacture three-dimensional structures but also create irregular curved surfaces. The equipment required for soft lithography is relatively simple and can be applied in a general laboratory environment. The graphic copying process is simple, provides high precision, and is repeatable. It is convenient, cheap, and suitable for general production and experimental environmental conditions, and low in cost.

2.4. Microfluidic Flow. Micro-nanoscale fluids are typical laminar flows. Wen Reynolds (Re) number is greater than or equal to 100. If there is a certain obstacle in the sidewall or the pipeline, an asymmetric structure will be formed, which will cause eddy currents and eddies in the flow field. When $100>\operatorname{Re} \geq 10$, it is challenging to form eddy currents in the microchannels of the planar structure, but influential eddy currents can still be generated in the microchannels of the three-dimensional asymmetric structure. For the case where the $\operatorname{Re}$ is extremely low, that is $\operatorname{Re}<10$, an asymmetric groove with a certain angle to the flow direction must be designed on the wall surface of the microchannel to create an anisotropic resistance to the fluid, causing local rotation of the fluid. Stretching can form a chaotic state and vortex. The most effective way to create eddy currents in a laminar flow with a low Re value is to prepare active agitating components at local locations in the fluid and then use external electric fields, magnetic fields, ultrasound, or pressure to control these components to form eddy currents.

Micro-nanofluids can be divided into single-phase flow (such as single liquid flow and single gas flow), two-phase flow, and multiphase flow (such as gas-liquid two-phase flow and liquid-liquid multiphase flow) according to their composition. Multiphase flow can be subdivided into a single component (multiphase, SCMP) and multicomponent (multiphase, MCMP) and can also be divided into mutual soluble multiphase flow and nonintermetallic dissolution phase flow.

2.5. Interface between Miscible and Immiscible Fluids. At the macroscopic scale, it is difficult to maintain a distinct interface between mutually soluble liquid fluids. However, under laminar flow conditions with low Re, fluids can only be mixed by interfacial diffusion. Therefore, even if the fluids are miscible, there is a clear interface in the middle when they contact each other. However, as the mutual contact time is extended, the interface is broadened and gradually blurred due to the diffusion in the longitudinal direction (along the fluid flow direction) and the lateral direction (perpendicular to the fluid flow direction). 


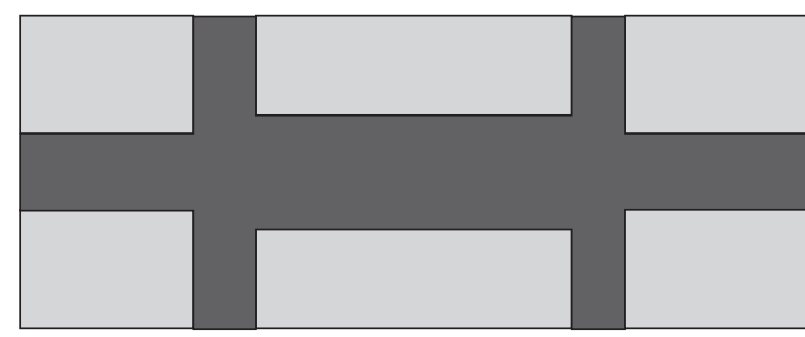

(a)

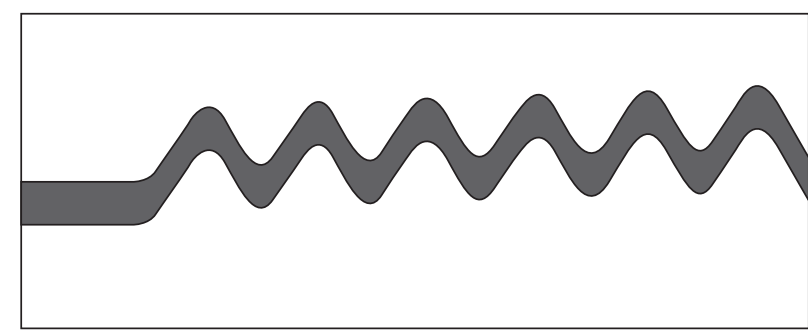

(b)

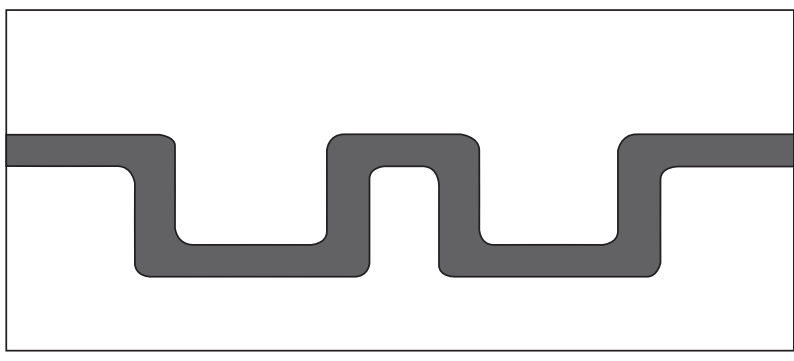

(c)

Figure 1: (a) Flat straight path; (b) two-dimensional curved main channel; (c) two-dimensional broken line main channel.

The interface of the immiscible fluid asks for the immiscible two-phase microfluid, and a clear interface can be maintained between the two phases for a long time, i.e., a socalled "pinning" interface is formed. However, the shape of the interface is affected by many factors such as fluid viscosity, interfacial tension, flow velocity, channel feature size, and channel inner wall state, forming stratified flow, wavy laminar flow, inclined interface laminar flow, droplet flow, plug flow, and ring shape. Interfacial control of miscible multiphase microfluidics is one of the key technologies in microfluidic technology. Under macroscopic conditions, the water-soluble and the organic phases are separated by gravity. However, for microfluidics, the interfacial tension between liquid-solid, liquid-gas, and liquid-liquid phases play a dominant role. The method of controlling fluid on a macroscopic scale is no longer applied again. Changing the microchannel structure, such as introducing the guiding structure $\mathrm{m} 1$ or using a microchannel with a specific shape cross section, can effectively change the interface morphology between the multiphase immiscible microfluidics. Surface chemical modification of the wall surface of the microchannel by PVP, PEO, PHEMA, ODS, etc., can make the interface morphology more stable and easy to control.

\subsection{Microfluidic Control: Parallel Porous Electric Drive Pump} Technology. Among the functional units required for microfluidic systems, microfluidic drive and control operating units are particularly important. Under the microsystem conditions, the influence of surface tension becomes very obvious. The conventional driving method of fluid volume flow is often not effective in micropipes in the engineering sense. Common microfluidic drive technologies include mechanical micropump technology and nonmechanical micropump technology based on electricity, light, magnetism, and highefficiency hybrid control of microfluidics.
Mechanical displacement micropump is the output part of mechanical kinetic energy, it converts mechanical energy into driven fluid. In this type of pump, the flow and pressure are easy to match with the microinfusion, and more emphasis is placed on flow matching. In general, the miniaturization and integration of the pump are not emphasized. A nonmechanical micropump is a continuous dynamic flow by converting or applying other forms of energy (electricity, light, magnetism, heat, etc.) to the driven fluid to have kinetic energy. Since it is generally a valveless structure, it is often called a continuous dynamic flow. Electric (direct) drive (fluid) pumps designed according to electrohydraulic principles are an essential category. In recent years, magnetic flow control technology has been developed to drive and control fluids by adding a magnetically magnetic nanoparticle medium to a fluid. The newly emerging novel lightdriven pump technology uses a light control method to control the fluid transport of a microsystem. It is a continuous flow pump technology with development potential. In addition, drive pump technology based on surface tension, gravity, and centrifugal force has also made rapid progress.

In this paper, the microfluidic device based on the segmented temperature sensor adopts the parallel porous electric drive pump technology.

A long time ago, the direct use of direct current or lowfrequency alternating current to drive fluids was the conventional way. However, now it has become a reality in the shape of an electrohydraulic power pump (EHD). The study began in the 1960s with an ion drag pump (IDP). IDP is an infusion pump that utilizes the migration movement of charged ions in a fluid under the action of an electric field. With the advancement of microfabrication technology, micro-IDPs etched on single crystal silicon have emerged. The micropump mainly has two etchings on the single-crystal silicon. The mesh channel is connected by 
electrodes (distance $350 \mathrm{ttm}$ ). Injecting the ionic fluid in the initial mesh causes the ions in the fluid to move under the action of the electric field to drive the fluid movement. The EHD can now be used on the chip to achieve fast mixing of fluids. The EKP developed according to the EHD principle mainly includes an electroosmotic flow- (EOF-) based electroosmotic pump (EOP) and an electrophoretic separation- (EPF-) based electrophoresis pump (EPP). Figure 2 is a schematic diagram of the chip EPP and EOP (the EPP gradually transitions to EOP when the microchannel size is reduced). Given its importance, we briefly outline its development history and characteristics here. EPP can also be called an open channel electroosmotic pump, mainly relying on the electrophoretic migration and/or electroosmotic flow principle of the medium in a microchannel under an electric field. It is a direct flow drive technology without a valve and mechanical parts. The driving of hi/rain to several $\mathrm{td} / \mathrm{min}$ flow in the microscale channel has the advantages of moderate flow, no pulsation of liquid flow, and easy integration. It has been widely used in the separation and analysis of capillary electrophoresis. Although various fluid-driven methods such as pressure, gravity, centrifugal force, and shear forces have appeared in the field of microsystems such as microfluidic chips, EPP is still the most basic liquid driving method at present. Many properties of microchannels in microfluidic chips are similar to those of capillaries. At the same time, with the expansion of microfluidic chip separation analysis research, microchannels in the chip often need to be made into more complex network structures, and there are different intersection areas. To realize the driving and control of fluid flow rate and flow direction in each channel, the easiest way is to adjust the different nodes in the microchannel network. An intelligent control electric drive with a voltage value to regulate the fluid, multiple electrode voltage programmable controls, and stable output is necessary. EOP occurs at the interface between the solution and solid. In order to effectively increase the surface area of the interface and make full use of EOF, it is easy to think of filling the microcapillary or microchannel with dielectric insulating microparticles. This is the packed bed electroosmotic pump (p-EOP). Simultaneously, the filler particles can also increase the countercurrent resistance of the EOF, thereby increasing the output pressure. Its working principle is similar to that of capillary electrochromatography (CEC) to achieve high-pressure microflow precise liquid manipulation. This type of pump is also called a highpressure electroosmotic pump. Typical characteristics of EOP are high pressure and low flow, and the general pressure range is from 0.1 to $50 \mathrm{MPa}$. The flow rate is from nanoliter to microupgrade. EOP was first reported around 2000 and is now very mature and has evolved into many forms, such as packed beds, parallel multichannel leads, monolithic columns, and microporous membranes. Schematic diagram of EPP and EOP on a glass-based chip is shown in Figure 2.

The parallel multichannel electroosmotic pump is a micropump based on the principle of electroosmosis, consisting of hundreds of parallel small-diameter

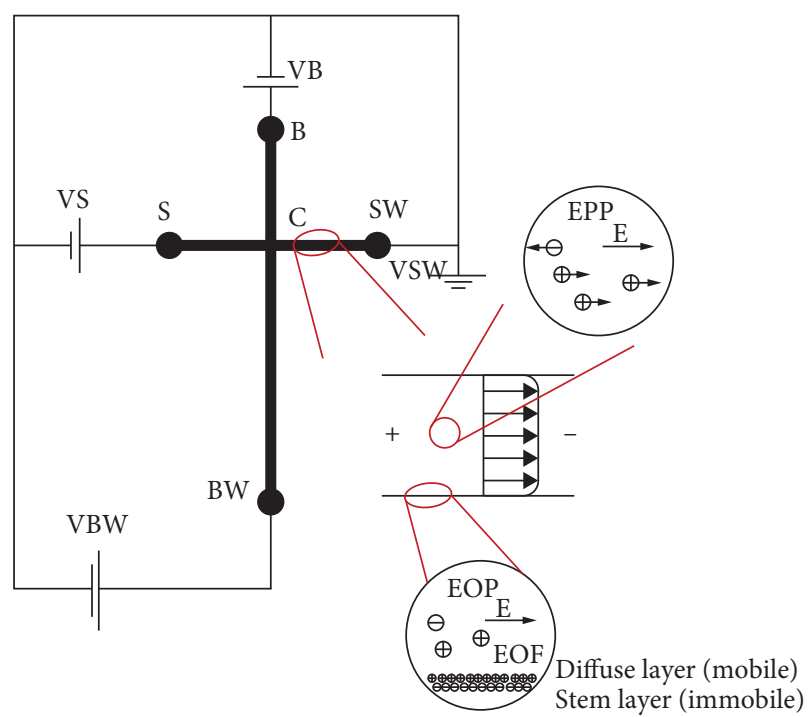

Figure 2: Schematic diagram of EPP and EOP on a glass-based chip.

microchannels or even nanochannels. It provides flow and pressure compatible with the microchip network structure for general analytical applications, i.e., $0.05 \mathrm{ltd} / \mathrm{min}$ flow and LQ arm backpressure. The small size makes it easy to achieve a multicomposite of a single pump. When the channel is a nanochannel, the pump is a nanochannel electroosmotic pump with the same function as p-EOP. The nanochannel electroosmotic pump has a high delivery pressure, and the pressure is in the $1-10 \mathrm{arm}$, and the flow rate is slightly upgraded. Essentially, since electrophoretic migration in nanochannels is difficult to participate in fluid delivery, this type of pump is a true electroosmotic pump and has the same principle as a capillary channel electroosmotic pump. The ideal electroosmotic pump should be battery-powered. How to effectively reduce the driving voltage is very important.

Compared with the primary electroosmotic pump, the three-stage electroosmotic pump provides an important idea that it is expected to develop a chip-based or miniaturized multistage electroosmotic pump as a microfluidic component, which has been successfully developed by researchers. A 10-stage electroosmotic pump is used for membranes; another effective method is to use a microchannel membrane as the medium for the electroosmotic pump. EKP is very important in microfluidic analysis systems because of the capillary effect, which is often the most efficient way to push fluids in micron-scale pipes (500 tan); it is easy to make as there are no moving parts and can be designed in very small spaces. It is within the volume and is compatible with the chip. EKP delivers buffer solutions and polar organic solvents that can be transported for most analytical chemistries, including liquid chromatography, capillary electrophoresis, and most fluids involved in microfluidic chips. EK has great potential for applications in capillary fluid chromatography, flow injection analysis, sequential flow injection analysis (and microfluid systems such as micro drug delivery). The main disadvantage of EKP is that it can only 
drive fluid media that can produce electroosmotic flow. Electric drive technology is a very efficient driving method. EKP has revolutionized the technology from conventional electrophoresis to capillary electrophoresis and played an irreplaceable role in the research field of life sciences. However, with the rapid development of micro-nanoscale component technology, limitations are also becoming more and more apparent. As shown in Table 1, EKP technology is very suitable for channel size in the range of $5 \mathrm{mbar}$ Tim, which is also the channel size range of capillary electrophoresis and microfluidic chips; the resistance is at a medium level, and the microfluidic efficiency of capillary electrophoresis and the microfluidic chip can be well realized by using a single current $\mathrm{DC}$ voltage $(\mathrm{Bu} 30 \mathrm{kV})$ and multichannel DC voltage $(50 \mathrm{kV})$ system using microcurrent $(500 \mathrm{~m})$. The drive and control are to avoid the extremely high Joule heat caused by the large size of traditional electrophoresis. However, the electric drive technology is not very suitable. The channel size is used in the range of $l$ to $50 \mathrm{tan}$. This size is also the range of channel size for singlemolecule research, especially for biological macromolecules. Because of the large equivalent resistance, even a short channel requires a higher driving voltage. Nanochannels can change the limitations of electric drive, and it is necessary to develop more effective drive methods in this area. Optical and magnetic drives have great advantages in solving such nanoscale fluid transport.

2.6.1. Function. The functions that a microfluidic device can perform are dependent on the transmission process in the fluid in the microchannels in the device. For example, a micropump port is a device that uses a different driving method to form an overall directional flow in a specific region of a microchannel. The microseparator utilizes the microparticle characteristics between different fluids. A device that effectively separates multiple particles or fluids, including a micromixer, which allows sufficient material exchange between the various parts of the microfluid to form a layer of mixture. A microreactor is a device that allows fluids that can react with each other to react effectively in the tiny space defined by microchannels or droplets to prepare specific substances. By integrating these devices and assembling them with external energy and signal input and output devices, a fully functional microfluidic system can be prepared for aerospace, medical, and agricultural applications, bioengineering, material processing, chemical industry, and many other fields. The limitation for the electrically driven technique is shown in Table 1.

\section{System Composition and Operation}

The measurement part is: the CCD camera is connected to the fluorescence microscope via the interface converter. The laser is fixed on the mirror arm of the fluorescence microscope so that the excitation light and the emitted light are in a confocal manner. The laser spot is $300 \mu \mathrm{m}$; the image capture card inserted in the peripheral component interconnect (PCI) slot of the personal computer saves the image data collected by the CCD in the hard disk and displays it on the screen at the same time; the temperature control part includes the Indium tin oxide (ITO) glass heater and the sticker attached to the ITO glass. Chip Pt resistance temperature sensor, adjustable constant current power supply for heating, multifunction data acquisition card for temperature acquisition and control signal input and output, and temperature control program were written by LabVIEW PID control module [14]. The temperature measurement and control system device is shown in Figure 3.

About 20 minutes before the start of the experiment, the ITO glass heating sheet was fixed on the $X 2 Y$ two-dimensional operating platform; the power of the experimental device was turned on, the temperature control and image acquisition procedures were run, the temperature control was adjusted to room temperature, and the heater was not heated, and the image acquisition program was set. The status is displayed in real time; the CCD camera is set to manual to ensure that the fluorescence images acquired at all temperatures have the same gain; the laser is turned on and shielded by a visor; the entire experimental system is preheated. Then, the channel of the microfluidic chip is filled with rhodamine $B$ aqueous solution ( $014 \mathrm{mmol}$ ), the chip is placed on the ITO heating sheet, the $X 2 Y$ two-dimensional operation platform is manually adjusted, and the display screen is observed so that the measured area is in the field of view, and the microscope is adjusted. The focal length makes the edges of the channel clearest. After the position and focal length were adjusted, the microchip was fixed with tape to prevent slippage and position during the experiment. At the beginning of the experiment, the fluorescence intensity image of the initial state of the rhodamine B solution (i.e., room temperature) was first collected. The heater temperature was controlled, and the fluorescence intensity image of the rhodamine B solution was collected at different temperatures. To prevent the rhodamine $B$ dye annihilation, the excitation light is blocked with a light barrier during the image capture.

To measure the temperature with the fluorescence intensity of the dye as the temperature indication, it is first necessary to determine the functional relationship between the temperature value and the dye fluorescence intensity value through experiments. The temperature calibration device is shown in Figure 4. A capillary tube with a length of about $20 \mathrm{~cm}$ removes about $2 \mathrm{~cm}$ of the polyimide outer cover as a detection window in the middle portion, attaches it to the ITO heating sheet, and uses a hard paper periphery of the detection window. The ring is looped just above the capillary, and the surrounding area is about $1 \mathrm{~cm}^{2}$. The PDMS prepolymer and the cross-linking agent are uniformly degassed and then poured into the enclosure. The effect of the PDMS film (about $400 \mu \mathrm{m}$ ) after curing is to fix the capillary on the ITO-heated glass sheet; on the other hand, it acts to conduct heat so that the temperature of the dye in the capillary is uniform. The platinum resistance temperature sensor is attached to the ITO heating sheet against the PDMS film. Control the temperature of the ITO heater, take a point from room temperature to $90^{\circ} \mathrm{C}$ every $5^{\circ} \mathrm{C}$, and equilibrate at least $10 \mathrm{~min}$ at each temperature point to ensure that the temperature sensor and the capillary-encapsulated rhodamine B fluorescent dye wrapped 
TABLE 1: Limitation for electrically driven technique.

\begin{tabular}{lcccc}
\hline & $\begin{array}{c}\text { Channel size } \\
(\mu \mathrm{m})\end{array}$ & $\begin{array}{c}\text { Suitable for electrically } \\
\text { driven }\end{array}$ & $\begin{array}{c}\text { Equivalent electric } \\
\text { resistance }\end{array}$ & Alternative \\
\hline $\begin{array}{l}\text { Conventional electrophoresis } \\
\text { Capillary electrophoresis, lab-on- }\end{array}$ & $\begin{array}{c}\text { more } \\
\text { chip, etc. }\end{array}$ & Fit, lower efficiency & Small & Syringe pump, etc. \\
$\begin{array}{l}\text { Single macromolecule analysis } \\
\text { Nanochannel research }\end{array}$ & $1-500$ & Fit, high efficiency & Middle & Electrically driven \\
pump & Large & Light-driven, etc. \\
\hline
\end{tabular}

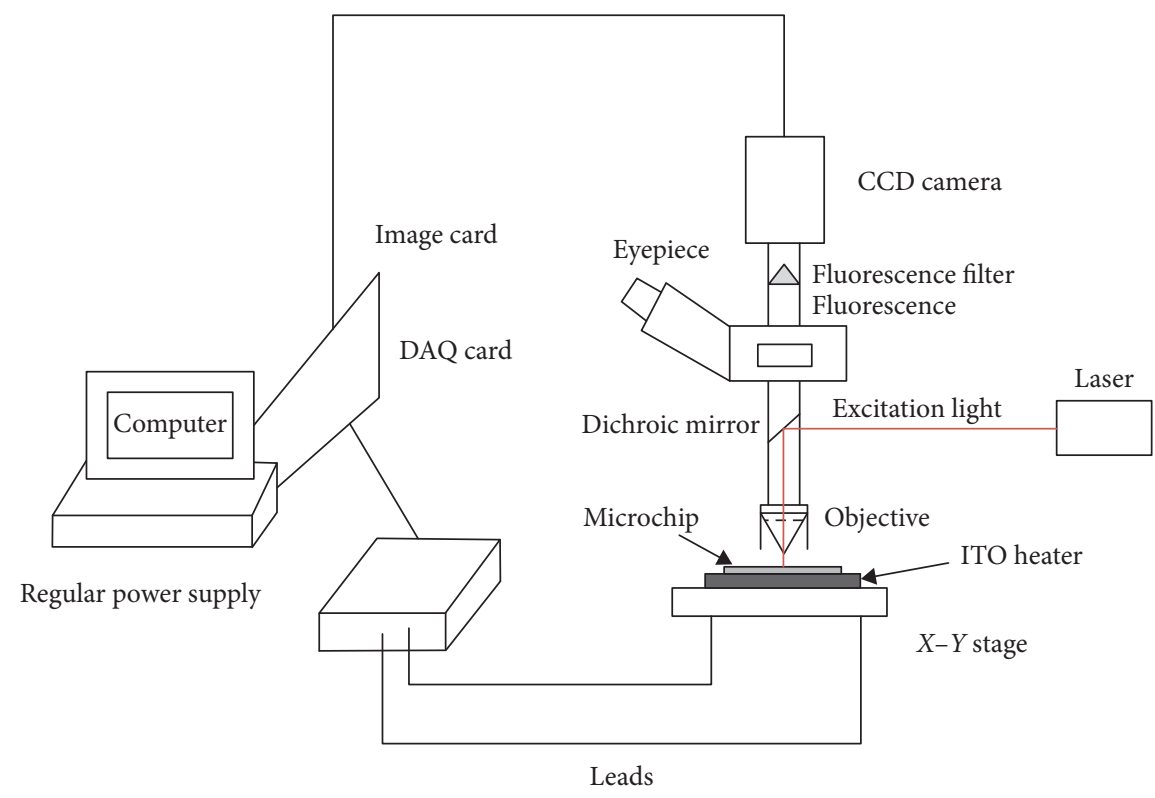

Figure 3: The temperature measurement and control system device.

in the PDMS film are in equilibrium with the heater temperature. At each temperature point, 25 fluorescence intensity images were continuously acquired, and $5 \times 5$ pixel points were averaged at the same position on each image to obtain the fluorescence intensity of rhodamine B dye corresponding to each temperature value. Image data filtering, averaging, and other processes are all programmed using LabVIEW software. The temperature calibration device is shown in Figure 4.

\subsection{Characteristics of ITO Film Glass of Heating Element and} Calculation of Its Resistance Value. The thickness of the ITO film is on the order of ns. Its physicochemical properties are stable. Its hardness is high. It has good adhesion to most substrates, and its resistance to acid, alkali, and organic solvents is strong. Moreover, the transmittance can reach $2 \times 10-4 \Omega 1 \mathrm{~cm}$ and $90 \%$ or more, respectively. It is used in the microfluidic chip heating system. It has the advantages of fast temperature rise, good stability, durability, and no optical detection window. In this experiment, commercial ITO film glass is used as the microfluidic system heater, which has many advantages. First, it is cheap and easy to obtain. Second, it is easy to process and can be directly cut into the desired shape for special and complicated graphics. It can be obtained by the etching method. The heater platform and the chip are separated structures, which will not be discarded due to scrapping of the chip; thirdly, the light transmittance of ITO is very good and does not affect the optical detection of the microfluidic system.

The ITO film is used as a heater. Its shape and resistance value have a great influence on the surface temperature distribution. The resistance value can be calculated according to its shape and size. As shown in Figure 5, the size of a rectangular ITO film is shown. The formula calculates the resistance:

$$
R=\frac{\rho \cdot L}{d \cdot w}
$$

where $\rho$ is the density of the ITO layer, $L$ is the length, $d$ is the thickness, and $w$ is the width of the ITO layer. The PID control algorithm and ITO film glass are used as heaters, and the temperature control accuracy is $\pm 011^{\circ} \mathrm{C}$. When the temperature rises, it usually reaches a stable value at $20-30 \mathrm{~s}$. The overshoot is small, generally not exceeding $015^{\circ} \mathrm{C}$, achieving ideal temperature control accuracy and temperature rise speed. A schematic diagram of the ITO layer is shown in Figure 5.

3.2. Temperature and Rhodamine B Dye Fluorescence Intensity Curve. The following formula can express the fluorescence intensity $I$ produced by the fluorescent dye after being excited: 


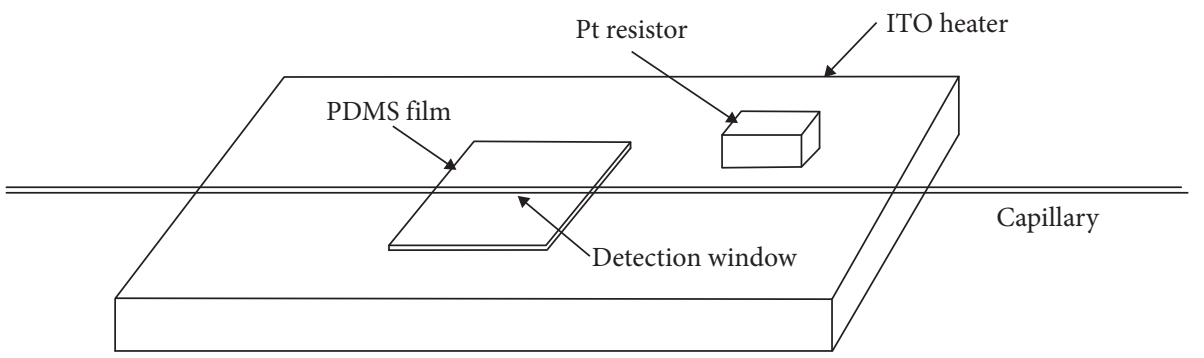

Figure 4: The temperature calibration device.

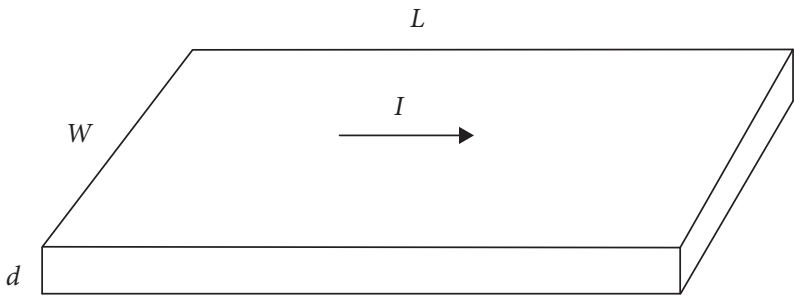

FIgURE 5: Schematic diagram of the ITO layer.

TABLE 2: The normalized experimental date with the temperature $\left({ }^{\circ} \mathrm{C}\right)$.

\begin{tabular}{|c|c|c|c|c|c|c|}
\hline Temperature & 20 & 25 & 30 & 35 & 40 & 45 \\
\hline Normalized intensity & 1.010 & 0.991 & 0.969 & 0.912 & 0.859 & 0.813 \\
\hline Temperature & 50 & 55 & 60 & 65 & 70 & 75 \\
\hline Normalized intensity & 0.736 & 0.672 & 0.580 & 0.511 & 0.442 & 0.368 \\
\hline Temperature & 80 & 85 & 90 & 95 & & \\
\hline Normalized intensity & 0.297 & 0.241 & 0.192 & 0.137 & & \\
\hline
\end{tabular}

$$
I=I_{0} \cdot c \cdot \varphi \cdot \xi
$$

where $I_{0}$ is the intensity of the excitation light, $c$ is the concentration of the fluorescent dye, $\varepsilon$ is the absorption coefficient, and $\varphi$ is the quantum efficiency. For some complex dyes, such as rhodamine $\mathrm{B}$, the change in fluorescence intensity due to quantum efficiency can reach $2 \%$ $\mathrm{K}^{-1}$. The absorption coefficient $\varepsilon$ has no obvious temperature characteristics, and its variation is less than $0105 \% \mathrm{~K}^{-1}$. Assuming that the quantum efficiency is inversely proportional to the absolute temperature over a certain temperature range, then

$$
\varphi=\frac{k}{T}
$$

where $k$ is the quantum efficiency constant and $T$ is the absolute temperature. From these two formulas, the following formula can be derived:

$$
I=\frac{k \cdot c \cdot I_{0} \cdot \xi}{T}
$$

Under the condition that the excitation light source intensity $I_{0}$ and the dye concentration $c$ are constant, there is

$$
T=\frac{K}{I}
$$

where $K=k \cdot I_{0} \cdot c \cdot \xi$.The temperature can be obtained by measuring the fluorescence intensity of the dye during measurement.
To measure temperature by this method, it is first necessary to calibrate the relationship between the intensity of the fluorescent dye and the temperature. In the actual temperature measurement process, the excitation light intensity $I_{0}$ and the dye concentration $c$ may be different from the temperature calibration. The change of the position of the detection point also causes the fluorescence collected by the CCD to change. In order to eliminate the influence of these factors on the temperature measurement, it is necessary to normalize the calibration curve between temperature and fluorescence intensity, that is, record first before each measurement. The temperature field at room temperature is used as the reference field (the parameter field contains the same parameter information in the same experiment process). Each measurement temperature field is divided by the reference field during the measurement process to obtain a relative change amount, thereby eliminating the parameter influences. After normalization, all factors affecting the measurement, including the measuring instrument, concentration, CCD position, the thickness of the liquid layer, and unevenness of the background of the measured area, are eliminated.

Table 2 shows the normalized experimental data and the temperature and fluorescence intensity (averaged from seven experimental curves on different dates).

Furthermore, the fitting formula is obtained by fitting the third-order polynomial (Figure 6). 


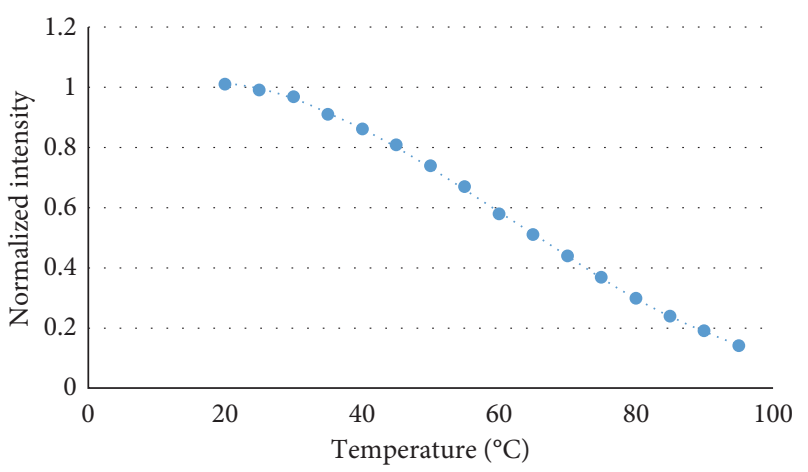

Figure 6: Temperature and fluorescence intensity curves.

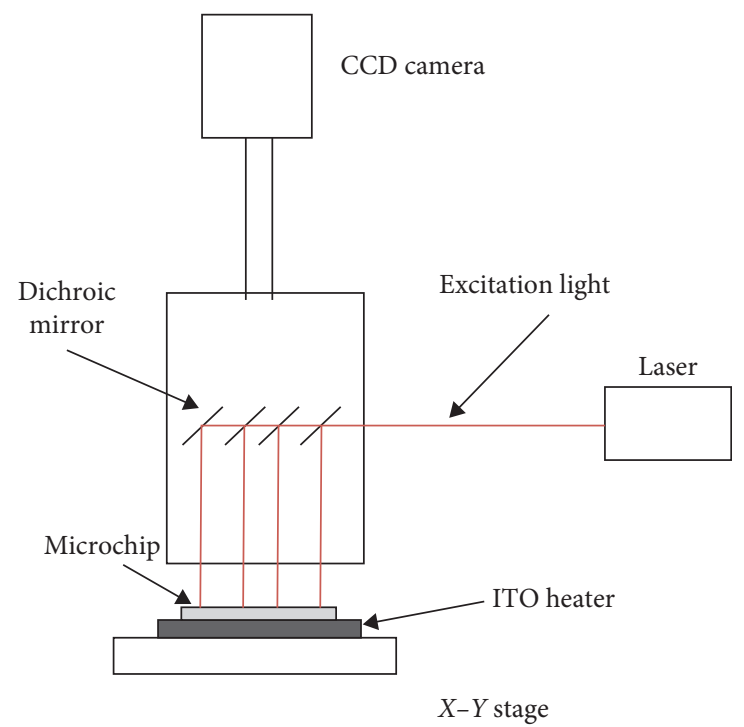

FIgURE 7: Concept map of segmented temperature sensor.

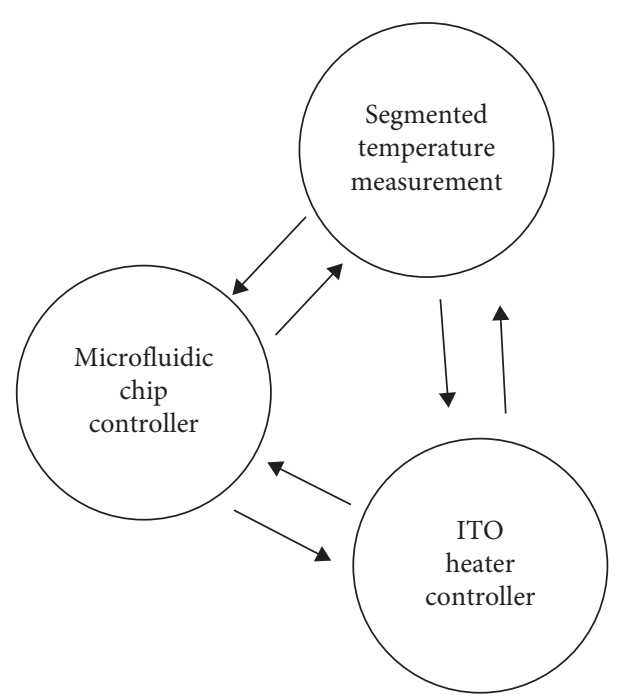

Figure 8: The schematic diagram of the control system. 


$$
I=A+B_{1} \cdot T+B_{2} \cdot T^{2}+B_{3} \cdot T^{3},
$$

where $A=0.9105, \quad B_{1}=0.0131, \quad B_{2}=-0.0005$, and $B_{3}=-0.000002$.

3.3. Segmented Implementation. The device uses a beam splitter to change the optical path and intensity of the excitation light source so that the device can obtain four beams of parallel light of equal intensity and irradiated on the microfluidic chip, thereby measuring the fluorescence intensity by the CCD camera. The concept map of a segmented temperature sensor is shown in Figure 7.

\section{Control System of the Microfluidic Device Based on the Segmented Temperature Sensor}

The microfluidic device based on the segmented temperature sensor designed in this paper can be used for segmental temperature measurement and temperature control of the solution in the microchannel of the glass microfluidic chip. The device uses a transparent indium tin oxide film glass as a heating element. It adopts a temperature control platform of a PID control algorithm. The system uses a CCD camera, a fluorescence microscope, and an image acquisition card to form a noncontact fluorescent indicator temperature measuring device, which realizes the measurement of the temperature distribution of the microfluid space and the temperature measurement with time, and controls the microfluid based on this. The schematic diagram is shown in Figure 8.

\section{Conclusion}

This paper proposes a microfluidic device based on the segmented temperature sensor. This device can be used for segmental temperature measurement and controlling the temperature of the solution in the microchannel of a glass microfluidic chip. The segmented temperature sensor-based microfluidic device control system is highly adaptable, simple in processing, and more flexible. The system uses a charged coupled device camera, a fluorescence microscope, and an image acquisition card to form a noncontact fluorescent indicator temperature measuring device. The device measures the temperature distribution of the microfluidic space with time and controls the microfluid. Moreover, the device has the advantages of simple structure, low cost, and convenient operation.

\section{Data Availability}

The datasets used and/or analyzed during the current study are available from the corresponding author on reasonable request.

\section{Conflicts of Interest}

The authors declare that they have no conflicts of interest.

\section{Acknowledgments}

The study was supported by "the Sponsored by Qing Lan Project (No. 10 of Official document from Jiangsu Education Department in 2020).”

\section{References}

[1] C. Abram, B. Fond, and F. Beyrau, "Temperature measurement techniques for gas and liquid flows using thermographic phosphor tracer particles," Progress in Energy and Combustion Science, vol. 64, pp. 93-156, 2018.

[2] B. Aneja, S. Singh, U. Chandna, and V. Maheshwari, "Review of temperature measurement and control," Ijarse Com, vol. 3, no. 1, pp. 33-40, 2014.

[3] L. Yu, W. Wang, X. Zhang, and W. Zheng, A Review on Leaf Temperature Sensor: Measurement Methods and Application. Computer and Computing Technologies in Agriculture IX, Springer International Publishing, New York, NY, USA, 2015.

[4] Y. Deng, Y. Zhang, S. Sun et al., "An integrated microfluidic chip system for single-cell secretion profiling of rare circulating tumor cells," Sci Rep, vol. 4, p. 7499, 2014.

[5] C. Dames, Encyclopedia of Microfluidics and Nanofluidics, Springer US, New York, NY, USA, 2008.

[6] Y. Xu, F. Jiang, J. Du, and D. Gong, "A cross-domain collaborative filtering algorithm with expanding user and item features via the latent factor space of auxiliary domains," Pattern Recognition, vol. 94, pp. 96-109, 2019.

[7] R. Samy, T. Glawdel, and C. L. Ren, "Method for microfluidic whole-chip temperature measurement using thin-film pol$\mathrm{y}$ (dimethylsiloxane)/rhodamine B," Analytical Chemistry, vol. 80, no. 2, pp. 369-375, 2008.

[8] J. Sakakibara and R. J. Adrian, "Whole field measurement of temperature in water using two-color laser-induced fluorescence," Experiments in Fluids, vol. 26, no. 1-2, 1999.

[9] Y. Xu, Y. Chu, F. Jiang, Y. Guo, and D. Gong, "SVMs classification based two-side cross domain collaborative filtering by inferring intrinsic user and item features," KnowledgeBased Systems, vol. 141, pp. 80-91, 2018.

[10] D. Dabiri and M. Gharib, "Digital particle image thermometry: the method and implementation," Experiments in Fluids, vol. 11 , no. 2-3, 1991 .

[11] H. M. Hiep, T. Nakayama, M. Saito, S. Yamamura, Y. Takamura, and E. Tamiya, "A microfluidic chip based on localized surface plasmon resonance for real-time monitoring of antigen-antibody reactions," Japanese Journal of Applied Physics, vol. 47, no. 2, pp. 1337-1341, 2014.

[12] X. Jiang, N. Shao, W. Jing, S. Tao, S. Liu, and G. Sui, "Microfluidic chip integrating high throughput continuousflow PCR and DNA hybridization for bacteria analysis," Talanta, vol. 122, pp. 246-250, 2014.

[13] Y. Xu, J. Yang, and Z. Xie, "Training SVMs on a bound vectors set based on Fisher projection," Frontiers of Computer Science, vol. 8, no. 5, pp. 793-806, 2014.

[14] B. Xu, W. Q. Du, J. W. Li et al., "High-efficiency integration of three-dimensional functional microdevices inside a microfluidic chip by using femtosecond laser multi foci parallel microfabrication," Scientific Reports, vol. 6, p. 19989, 2016.

[15] L. L. Josephson, W. J. Galush, and E. M. Furst, "Parallel temperature-dependent microrheological measurements in a microfluidic chip," Biomicrofluidics, vol. 10, no. 4, p. 32, 2016.

[16] N. Inomata, M. Toda, M. Toda, and T. Ono, "Microfabricated temperature-sensing devices using a microfluidic chip for 
biological applications," International Journal of Automation Technology, vol. 12, no. 1, pp. 15-23, 2018.

[17] Y. Xu, D. Zhan, L. Liu, H. Lv, L. Xu, and J. Du, "A privacypreserving cross-domain healthcare wearables recommendation algorithm based on domain-dependent and domainindependent feature fusion," IEEE Journal of Biomedical and Health Informatics, vol. 5, 2021.

[18] G. Bonciolini, A. Demello, D. Vigolo, and E. Sciubba, "Microfluidic in-chip temperature control via the heat of mixing release," in Proceedings of the ECOS 2016, International Conference on Efficiency, Cost, Optimisation, Simulation and Environmental Impact of Energy Systems, Portorož, Slovenia, June 2016.

[19] X. Y. Chen, T. Y. Li, S. Zhang et al., "Research on optimizing parameters of thermal bonding technique for pmma microfluidic chip," International Polymer Processing, vol. 32, no. 3, pp. 394-398, 2017.

[20] M. Yu, T. Quan, Q. Peng, X. Yu, and L. Liu, "A model-based collaborate filtering algorithm based on stacked AutoEncoder," Neural Computing and Applications, vol. 9, 2021.

[21] M. Olivero, A. Vallan, R. Orta, and G. Perrone, "Single modemultimode-single mode optical fiber sensors: review and application to temperature measurements using a bend-insensitive fiber," in Proceedings of the Instrumentation and Measurement Technology Conference, pp. 1-5, IEEE, Turin, Italy, May 2017.

[22] R. Wang, Y. Xu, R. Wang et al., "A microfluidic chip based on an ITO support modified with ag-au nanocomposites for sensors-based determination of melamine," Microchimica Acta, vol. 184, no. 1, pp. 1-9, 2017. 